\title{
The Building of Pulsed NQR/NMR Spectrometer
}

\author{
Preeti Hemnani $^{1}$, A. K. Rajarajan ${ }^{2}$, Gopal Joshi ${ }^{3}$, S. V. G. Ravindranath ${ }^{4}$ \\ ${ }^{1}$ Homi bhabha National Institute, Mumbai, India \\ ${ }^{1}$ S.I.E.S Graduate School of Technology, Mumbai, India \\ ${ }^{2,3,4}$ Bhabha Atomic Research Centre, Mumbai, India
}

\begin{tabular}{l} 
Article Info \\
\hline Article history: \\
Received Jan 2, 2018 \\
Revised Mar 14, 2018 \\
Accepted Mar 28, 2018 \\
\hline Keyword: \\
ALE \\
ANC \\
DDS \\
FPGA \\
NMR \\
NQR
\end{tabular}

\begin{abstract}
NQR spectrometer designed is composed of four modules; Transmitter, Probe, Receiver and computer controlled (FPGA \& Software) module containing frequency synthesizer, synchronous demodulator, pulse programmer and display. The function of the Transmitter module is to amplify the RF pulse sequence to about $200 \mathrm{~W}$ power level into the probe $(50 \mathrm{Ohm})$ which is a parallel resonance circuit with a tapped capacitor. The probe excites the nucleus and picks-up the signal emitted from the nuclei. The nuclear signal at the same frequency as the excitation, which is typically in the range of a few microvolts is amplified, demodulated and filtered (1 kHz to $100 \mathrm{kHz}$ ) by receiver module. ${ }^{14} \mathrm{~N}$ NQR, ${ }^{1} \mathrm{H}$ and ${ }^{2} \mathrm{H}$ NMR signals are observed from the spectrometer.As the SNR of NQR signal is very low, NQR signal processing based on Adaptive Line Enhancement is presented.
\end{abstract}

Copyright $(0) 2018$ Institute of Advanced Engineering and Science. All rights reserved.

Corresponding Author:

Preeti Hemnani,

S.I.E.S Graduate School of Technology,

Nerul Navi Mumbai,

India.

Email: preetiahemnani@gmail.com

\section{INTRODUCTION}

Nuclear Quadrupole Resonance (NQR) is a branch of radio frequency spectroscopy which measures the interaction between the nuclear electrical quadrupole moment and electronic field gradient produced by electronic charge distribution about the nuclei in a solid state material. Radio frequency field is applied to a sample placed inside a coil in a pulsed mode. These RF pulses cause nuclei to resonate and absorb energy from this RF field. During the 'off' period of the pulse these nuclei induce an electric potential in a receiver coil. NQR is used to detect specific chemical compounds including explosives [1].

Nuclear Magnetic Resonance (NMR) is a RF technique that is able to detect any compound by sensing the excited resonance signals from atomic nuclei having non-zero spin. NQR is similar to NMR but the only difference is NMR needs a large magnetic field, and due to this its application in field is limited, and therefore most of NMR based explosive detection systems are developed for luggage screening. NQR detection only works for the quadrupolar nuclei with spin, I $>1 / 2$ i.e. the nuclei need to have quadruple moments. Most of the explosive substances are typically rich in ${ }^{14} \mathrm{~N}$ nuclei with $\mathrm{I}=1$ and thus ${ }^{14} \mathrm{~N}$ NQR is a suitable technique for explosive detection. The NQR detection starts with exciting ${ }^{14} \mathrm{~N}$ using RF radiation followed by detecting the radiation resulting from the decay of the excited ${ }^{14} \mathrm{~N}$ nuclei to their ground state. The RF radiation is produced by passing RF power through coil in resonance circuits which is also used for its detection. Since the resonance frequency depends on the crystalline and molecular environment of ${ }^{14} \mathrm{~N}$ in the explosive, the NQR spectra are unique for a given material and are not influenced by the interference of other nitrogen containing materials during the NQR measurements. This makes it attractive as non-invasive technique to detect explosives in landmines and screening baggage for explosives at airport [2]. The detection of radiation resulting from the decayed ${ }^{14} \mathrm{~N}$ nuclei to their ground state is then performed. The relaxation of 
nuclei termed Free Induction Decay (FID) induces a RF signal in the pick-up (excitation) coil. The NMR/NQR spectrum is obtained by fourier transforming the FID.

NQR spectrometers as detection systems are developed in two aspects, which are hardware configuration and NQR signal processing techniques. The conventional NQR configuration was large and consisted of standalone analog devices [3]. The challenge in the configuration was to reduce the size of the instrument and also to detect the weak signals by constructing a specialized sensitive spectrometer system using optimum experimental techniques. To overcome the challenge and gain the mentioned functionalities, Field Programmable Gate Array (FPGA) based NQR spectrometer is designed and developed resulting in a compact and completely programmable instrument with high sensitivity and suitable for applications like mine detection and non-invasive compound identification etc. Moreover, by adding a permanent magnet of uniform field one could carry out NMR as well which, can be a very useful tool for quantitative analysis of trace elements like Tritium and deuterium etc.

Since NQR signal is weak $(-100 \mathrm{dBm})$, for which rigorous and continuous research is required to develop post processing algorithms for NQR signal processing that are capable of enhancing the detection performance.

\section{CHALLENGES IDENTIFIED}

One of the most common challenge of $\mathrm{NQR}$, when compared to other detection techniques is the NQR signals are very weak (as low as $-100 \mathrm{dBm}$ ) which makes its SNR low. The SNR has to be improved such that the signal can be observed on scope (minimum 10dB). To improve SNR, many repetitions of the experiment are necessary. The most common method is to use repeated single RF pulse and acquire NQR signal after each pulse, but the acquisition depends on relaxation time of the nuclei. For most of the explosives, the relaxation times are very long which leads to long detection times [1]. For ${ }^{14} \mathrm{~N}$ containing compounds, the NQR signal frequency is in the range of $500 \mathrm{KHz}$ to $6 \mathrm{MHz}$, which overlaps the frequency band of AM radio. Thus, the NQR signals are always susceptible to Radio Frequency Interference (RFI) [1] which may severely corrupt the detection performance. The strong RFI from AM radio stations can lead to low Signal to Interference Ratio (SIR) and poor detection performance. While it is objectively straight forward to shield the pick-up coil in the laboratory, it may not be a viable option in the field.

Also, the uncertain amplitude is a common issue for NQR signals detected in the field. This has enticed the attention of several researchers in developing post processing algorithms for NQR signal processing and enhancing its detection performance. The challenge in NQR signal detection is to reduce the effect of RFI and to detect the weak NQR signal from background noise. It is evident that to fulfill this complex detection system, they must be equipped with a versatile computational platform.

\section{DEVELOPMENT OF NQR/NMR SPECTROMETER}

The first part of this work is to develop a FPGA based ${ }^{14} \mathrm{~N}$ NQR spectrometer [4]. The key strategy for developing this spectrometer is to fully exploit a FPGA as FPGA chip is composed of large number of programmable logic gates, in which digital circuits can be built with hardware description languages such as VHDL and Verilog. FPGA circuits can be reused after their development as per the requirement, also the individual circuits in FPGA run concurrently with reconfigurable capability. The FPGA based spectrometer, therefore, employs a single FPGA to perform all the digital tasks required for NMR/NQR spectrometer including pulse programmer (PP), direct digital synthesizer (DDS), digital receiver composed of quadrature demodulator, a low pass Finite Impulse Response (FIR) filter and PC interface for data transfer. The digital component is compact with high sensitivity and is suitable for applications like mine detection and noninvasive compound identification etc. Also, the analog peripheral components such as pre-amplifier, cross diodes and quarter wave transformer (used for isolation of transmitter and receiver) and Probe (Coil designing along with resonance circuits) were designed and developed as part of the current research. Figure 1 shows the block diagram of the NQR spectrometer. 


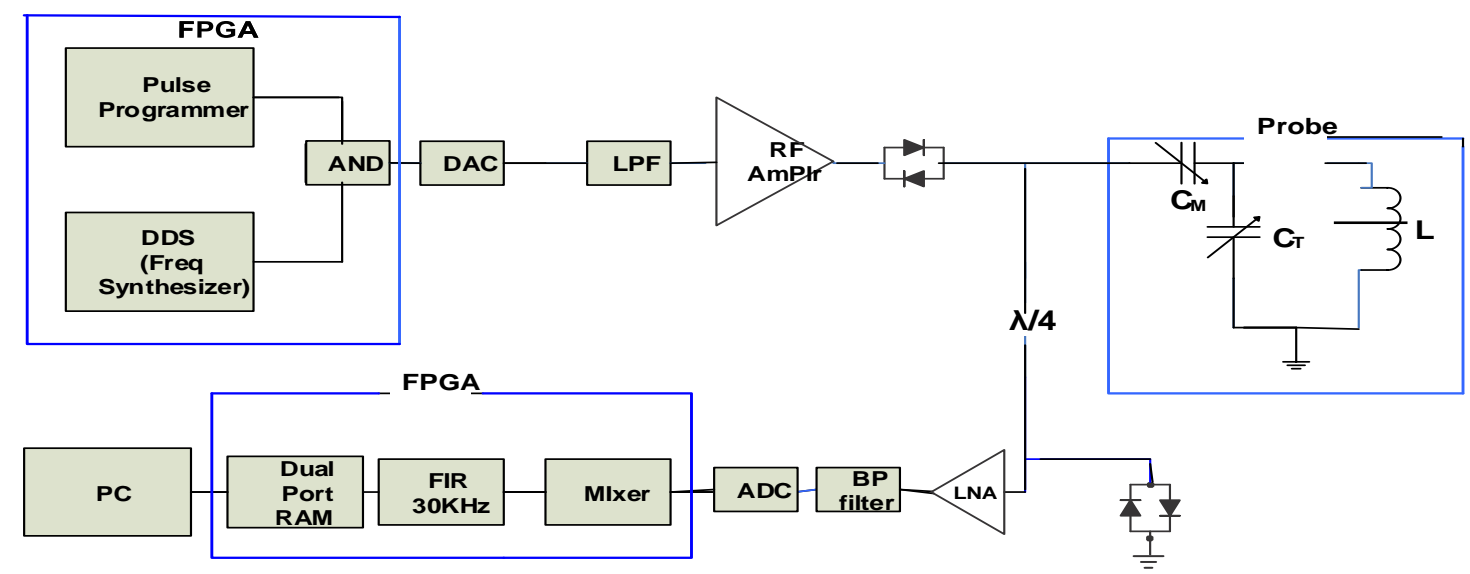

Figure 1. Block Diagram of designed FPGA based NQR spectrometer

\subsection{Specifications of the instrument}

Specifications of the NQR instrument: Frequency between $0-6 \mathrm{MHz}$; pulse lengths between 1-500 $\mu \mathrm{s}$; RF pulse power up to 200 watts and acquisition time of $50 \mathrm{~ms}$. The developed spectrometer consists of:

a. Transmitter, which consists of RF power amplifier to provide $13.5 \mathrm{MHz}$ RF signal of power $200 \mathrm{~W}$.

b. Probe which can be tuned to observe ${ }^{14} \mathrm{~N},{ }^{1} \mathrm{H},{ }^{2} \mathrm{H}$ signals.

c. Receiver, which consists of preamplifier (80dB gain), band pass filter (cut off frequency $4.64 \mathrm{MHz}$, Band Width- $500 \mathrm{kHz}$ ), ADC and DAC.

d. FPGA module, which consists of pulse programmer (pulse length $=1-500 \mu \mathrm{s}$, Acquisition time $0-10 \mathrm{~s}$ ) DDS (0 -6MHz) [5], gate, quadrature demodulator, FIR low pass filter (cut off $30 \mathrm{kHz}$ ).

e. Computer controlled module, which is used for command/data transfer.

\subsection{Experimental procedure}

First, a set of RF excitation pulses are applied to the nuclei placed in a coil. The resulting signal which starts at the excitation completion cycle is picked up by the same coil. The RF is generated by DDS and is then modulated by the pulses from pulse generator to obtain RF pulses. These RF pulses are then amplified by a RF amplifier for delivering an instantaneous power of up to $200 \mathrm{~W}$ to the probe circuit. The probe circuit is configured (as shown in Figure 1) such that the impedance of circuit, close to the resonance frequency, is $50 \Omega$ when the capacitors are appropriately adjusted. By matching the impedance of the probe circuit to characteristic impedance of the coaxial cables carrying the signal and other components in the circuit (amplifiers), all of the power generated by the power amplifier is transferred to the probe circuit, and the signal generated by the probe circuit is efficiently transferred to the receiver module. The FID generated is a weak signal $(-100 \mathrm{dBm})$ in the coil, which is amplified by the low noise amplifier before being mixed with a signal from DDS. This mixed down signal, which is at a frequency equal to the difference between DDS frequency and frequency of the precessing spins, is filtered. The application program is written in Labview/CVI through which all the communication to FPGA is done via cPCI bus. GUI developed in CVI for NQR/NMR spectrometer) is shown in Figure 2.

There are two popular methods of observing NQR/NMR signals are: a) observe FID using a single pulse excitation. b) Observe spin echo using a double pulse sequence. The choice of the sequence depends mainly on the relaxation time $\left(T_{2}^{*}\right)$ of the sample. For a long relaxation time of milliseconds $(0.3-3 \mathrm{~ms})$ as in the case for ${ }^{14} \mathrm{~N}$ signal from $\mathrm{NaNO}_{2}$, a single pulse sequence is appropriate and for a short relaxation time as in the case of ${ }^{1} \mathrm{H}$ and ${ }^{2} \mathrm{H}$ double pulse sequence is used to observe a spin echo.

In first method the excitation signal is high power RF pulse signal at resonant frequency of material. The width of RF pulse is denoted by $\tau$. This RF pulse is applied to a coil that contains the sample to induce an oscillating magnetic field $\left(B_{1}\right)$, The magnetic field tilts the nuclei from the reference axis by an angle $\theta$ which depends on power and duration of RF pulse.

$$
\Theta=\gamma \mathrm{B}_{1} \tau
$$


Then during the process that the nuclei attain the equilibrium state, FID can be detected on the same coil or separate receiving coil. The time constant by which nuclear spin systems returns back to equilibrium state is called spin -lattice relaxation time $\left(\mathrm{T}_{1}\right)$.

When nuclei precess after the RF pulse is applied, the small frequency differences from different spins lead to phase differences between the signals due to which they add up incoherently and cancel out, which occurs earlier than the spins actually return to the equilibrium state. The time constant for FID decay is denoted by $\mathrm{T}_{2}{ }^{*}$. The strength of FID signal depends on the flip angle $\Theta$. Highest NQR signal is achieved when $\Theta=\pi / 2$ thus the pulse width that results to maximum FID signal power is referred as $\pi / 2$ pulse width. The FID signal induced by one excitation RF pulse is very weak and thus SNR of the signal is very low to make an accurate detection. Thus most of the NQR detection systems actually perform the detection by repeating the detection cycle many times and NQR FIDs are acquired in time intervals between RF pulses. The numbers of NQR FIDs are averaged to increase the SNR so that the detection accuracy can be improved. The nuclei have to begin with equilibrium state for each individual detection cycle, so the spacing between the pulses $\left(\mathrm{t}_{\mathrm{gap}}\right)$ has to be no longer that $5 \mathrm{~T}_{1}$.

The magnetic moment of nuclei precessed with the characteristic angular frequency which is called Larmour frequency, is given by

$$
\omega_{o}=\gamma B_{o}
$$

where $\gamma$ is gyrometric ratio of nuclei and $B_{o}$ is applied magnetic field. The power requirements of the RF transmitter used in an NMR experiment/NQR experiment depend not only on $\gamma$ of the nucleus but also on the sample coil geometry. It has been shown that $\mathrm{H}_{1}$ power in the sample coil is given by [6].

$$
H_{1}=3\left(P Q / v_{o} V\right)^{1 / 2}
$$

where $P$ is transmitter power in watts; $Q$ is the quality factor of the probe, $v_{o}$ is resonance frequency in $\mathrm{MHz}$ and $\mathrm{V}$ is the volume of the sample coil in $\mathrm{cm}^{3}$.

The other type of NQR signal that can be generated is called spin echo. It requires two RF pulses with different phases to produce an echo signal. FID and spin echo signals are shown in Figure 3 . The mechanism of generating echo signals can simply be interpreted as refocusing the out of phase FIDs. After application of $1^{\text {st }} 90^{\circ} \mathrm{RF}$ pulse an FID is generated. As the ${ }^{14} \mathrm{~N}$ spins have slightly different NQR frequencies, the spins are soon out of phase and resultant FID decays very fast as expected. Then a $2^{\text {nd }} 180^{\circ}$ RF pulse is applied after a spacing of $t_{\text {gap. }}$. Thus all out of phase ${ }^{14} \mathrm{~N}$ spins are flipped by $180^{\circ}$ which makes the spins start to precess in opposite direction. Thus the spins are refocused at time $t_{\text {gap }}$ after second pulse and spin echo is radiated.

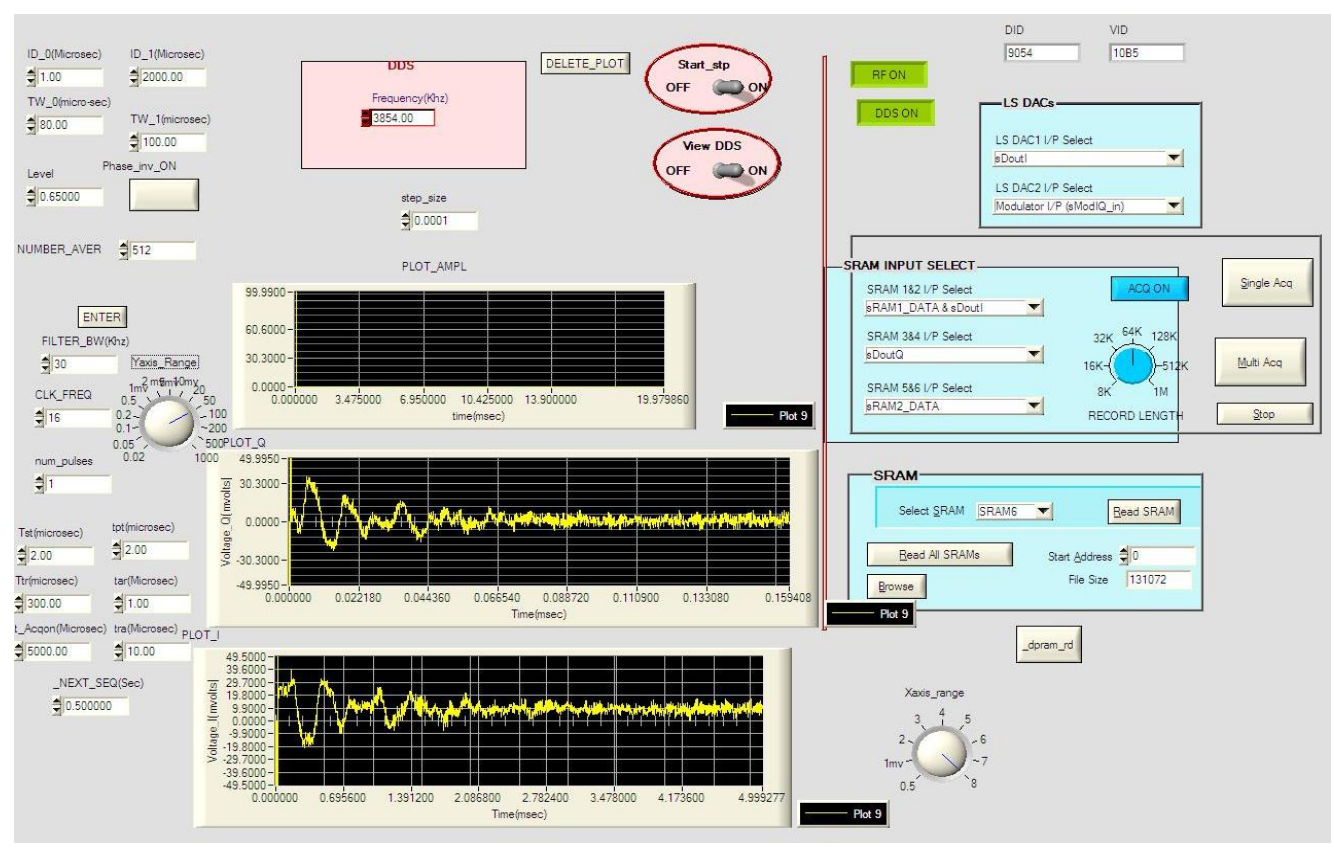

Figure 2. Application and GUI developed in Labview/CVI for NQR/NMR spectrometer 


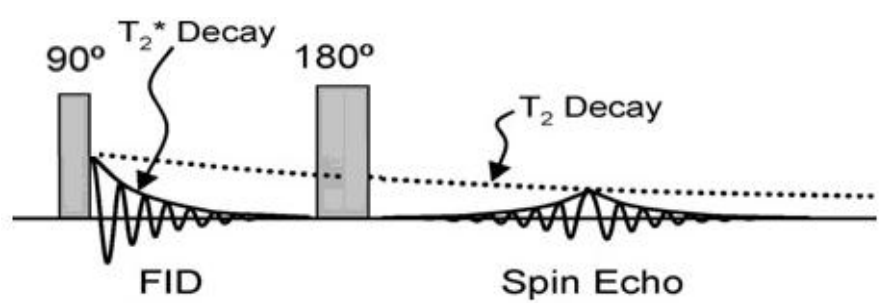

Figure 3. FID and Spin Echo in time domain

\section{RESULTS}

The stable nitrogen ${ }^{14} \mathrm{~N}$ has natural abundance of $93.6 \%$ and nuclear spin $\mathrm{I}=1$ with its associated nuclear electrical quadruple moment. The ${ }^{14} \mathrm{~N} N Q R$ transitions in various solids fall in the frequency range 0 to $6 \mathrm{MHz}$ [7]. Hence ${ }^{14} \mathrm{~N}$ was chosen for the design. ${ }^{14} \mathrm{~N}$ NQR signal from sample of $20 \mathrm{~g}$ of $\mathrm{NaNO}_{2}$ was observed at a frequency of $4.64 \mathrm{MHz}$. With the addition of permanent magnets ${ }^{1} \mathrm{H}$ and ${ }^{2} \mathrm{H}$, NMR signal from $\mathrm{H}_{2} \mathrm{O}$ and $\mathrm{D}_{2} \mathrm{O}$ was detected. Observation of Proton NMR is easy as the strength of this signal is strong as compared to ${ }^{14} \mathrm{~N}$ NQR signals therefore to standardize our spectrometer, we have observed ${ }^{1} \mathrm{H}$ NMR.

\section{1. $\mathrm{NQR}$ signal from $\mathrm{NaNO}_{2}$}

Figure 4 shows the ${ }^{14} \mathrm{~N}$ NQR signal in $20 \mathrm{~g}$ of Sodium Nitrite. The observation frequency is 4.642 $\mathrm{MHz}$, whereas the signal is off resonant by about $2500 \mathrm{~Hz}$ resulting in oscillatory FID. RF excitation pulse length of $20 \mu \mathrm{s}$ and power of $120 \mathrm{~W}$ was used for excitation of the nuclei. The pulse length is selected such that it is a $\pi / 2$ pulse, to get maximum amplitude of FID.

First, the power was kept constant and pulse width was increased. Then pulse width was kept constant and the power was increased. The resultant value of $20 \mu$ s as pulse width and Power of $120 \mathrm{~W}$ was obtained which matches with Equations (1) and (3) approximately. The entire cycle is repeated after every $0.5 \mathrm{~s}$ (large compared to the $\mathrm{T}_{2}{ }^{*}$ of $0.7 \mathrm{~ms}$ ). The signal strength is at the expected level of $6 \mu \mathrm{V}$. The NQR signal shown in Figure 4 is averaged for 256 times.

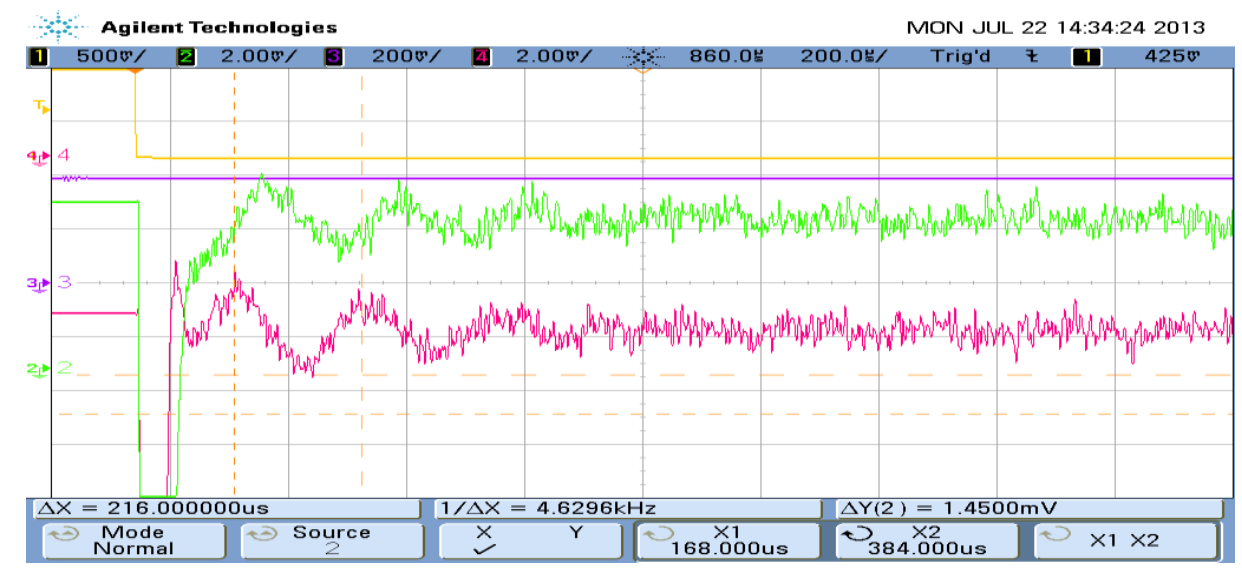

Figure $4 .{ }^{14} \mathrm{~N} N Q R$ signal from $\mathrm{NaNO}_{2}$

\section{2. ${ }^{1} \mathrm{H}$ NMR signal}

The NQR spectrometer was modified to NMR spectrometer by placing the coil in a permanent magnet. At the design frequency of $6 \mathrm{MHz}$, proton NMR can be obtained at a magnetic field of $2.35 \mathrm{kG}$. As a horse shoe magnet of $1.35 \mathrm{KGauss}$ was available, the spin echo experiment was carried out with $\mathrm{H}_{2} \mathrm{O}$ to observe the ${ }^{1} \mathrm{H}$ NMR signal. A small quantity of ferric nitrate is added for reducing $T_{1}$ of pure water.

Figure 5 shows the ${ }^{1} \mathrm{H}$ spin echoes from $\mathrm{H}_{2} \mathrm{O}: \mathrm{FeNO}_{3}$. The observation frequency is $5.765 \mathrm{MHz}$ (from Equation (2)). We used two RF pulses (spin echo sequence) with pulse widths $t_{1}=5 \mu \mathrm{s}, t_{2}=10 \mu \mathrm{s}$ and spacing between pulses is $t_{\text {gap }}=200 \mu s-650 \mu \mathrm{s}, \mathrm{RF}$ excitation power was about $70 \mathrm{~W}$. The power and pulse width was selected (from Equations (1) and (3)) such that $t_{1}$ is a $\pi / 2$ pulse. At resonance, voltage 
across capacitor reaches a value of Q times the applied voltage, which was exceeding the rating $(5 \mathrm{kV})$ of the capacitor and thus resulting in sparks in the capacitors. The entire cycle was repeated after every $0.5 \mathrm{~s}$. The signal strength was $50 \mu \mathrm{V}$. The trace shown in Figure 6 is the signal after averaging for 256 times. The two pulse experiment was performed for measuring $T_{2}^{*}$ by varying the spacing between the pulses. $T_{2}^{*}$ for ${ }^{1} \mathrm{H}$ in water is calculated from Figure 5 as $367 \mu$ s.

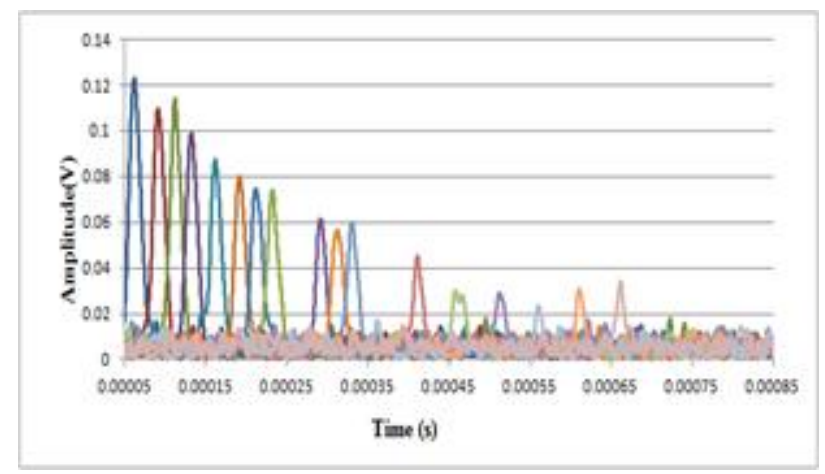

Figure 5. NMR signal of ${ }^{1} \mathrm{H}$ in $\mathrm{H}_{2} \mathrm{O}$

\section{3. ${ }^{2} \mathrm{H}$ NMR signal}

The $\gamma$ for deuterium is $41.065 \times 10^{6} \mathrm{rad} /$ Tesla, hence the magnet used for observing proton cannot be used with same setup, thus the magnet with a magnetic field of $5.85 \mathrm{kG}$ was used here to observe the spin echo of deuterium from $\mathrm{D}_{2} \mathrm{O}$. The deuterium signal is shown in Figure 6. The observation frequency is $3.855 \mathrm{MHz}$ (from Equation (2)). $t_{1}=5 \mu \mathrm{sec}, t_{2}=10 \mu \mathrm{s}, \tau=2 \mathrm{~ms}$.

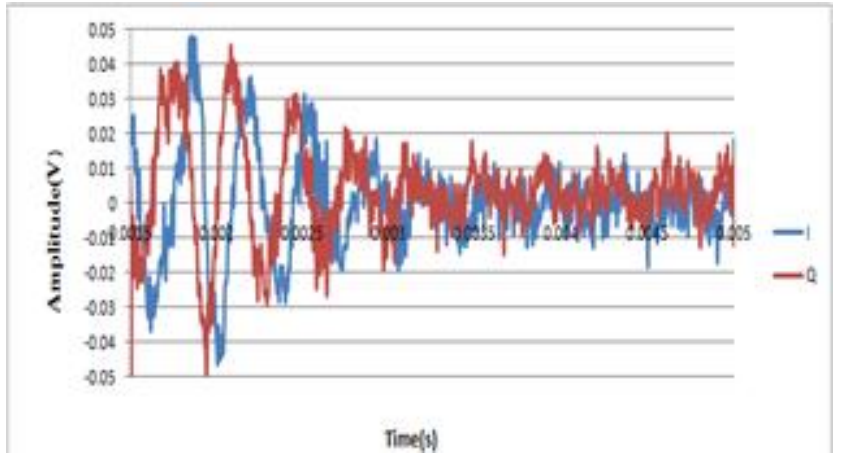

Figure 6. ${ }^{2} \mathrm{H}$ NMR signal from $\mathrm{D}_{2} \mathrm{O}$

\subsection{Post processing of NQR signals}

In field, the time required to detect the NQR signal is an important parameter. While, the main challenge for NQR techniques is the extremely poor signal to noise ratio (SNR), variations in the other parameters like temperature could also influence the signal strength and position. Under such conditions, improving SNR by averaging response to repetitive excitations could prove futile. The rate at which RF pulse has to be repeated depends on physical parameters of nuclear relaxation, which are, $T_{1}$ and $T_{2}$. In practice we can apply a pulse sequence of length $T_{2}$ and repeat the pulse sequence every $T_{1}$. For most of explosives the relaxation times are very long which lead to long detection times [1].

The signal shown in Figure 5 is ${ }^{14} \mathrm{~N}$ NQR signal observed from $\mathrm{NaNO}_{2}$. The signal obtained after one pulse was very weak so the SNR was improved by accumulation of 1024 times.

As we cannot shorten the relaxation times, much effort has been put into increasing the sensitivity of receiver and improving signal detection technique. To improve SNR per unit time several other techniques have been used i.e. FIR filter [8], wavelet transform [9], and adaptive filter [10]. FIR filter is generally based on prior knowledge of signal. Adaptive filter algorithms are used in this work as these do not need prior knowledge of signal and also parameters of adaptive filter changes to meet the optimization parameters. 
An adaptive filter is a self-modifying digital filter that adjusts its coefficients for minimizing an error function. This error function, also referred as the cost function, is a distance measurement between the reference or desired signal and the output of the adaptive filter. Adaptive Noise Cancellation (ANC) and Adaptive Line Enhancement (ALE) are two adaptive filtering systems with similar mechanisms but slightly different designs [11], [12]. ANC has two sensors to receive target signal and noise separately. The primary signal $d(n)$ consists of signal and noise $N_{o}$ together and a reference signal $x(n)$ and noise $N_{I}$ which are not correlated to the signal but correlated to noise $\mathrm{N}_{\mathrm{o}}$. The reference signal passes through the adaptive filter and output $y(n)$ is produced as approximately as $N_{o}(n)$. The filter readjusts its coefficients continuously to minimize the error between $\mathrm{N}_{\mathrm{o}}(\mathrm{n})$ and $\mathrm{y}(\mathrm{n})$ during this process. The output $\mathrm{y}(\mathrm{n})$ is subtracted from the primary input to produce the system output $e=S+N_{o}-\mathrm{y}$, which is a filtered signal. e(n) provides the system control signal and updates the adaptive filter coefficients, which minimizes residual noise.

ALE only requires a single sensor and delay to produce a delayed version of $\mathrm{d}(\mathrm{n})$, which decorrelates the noise while leaving the target signal component correlated. This is because noise is a broadband signal which is not correlated to the previous sample values unlike a narrowband signal. The delay causes decorrelation between the noise components of the input data in two channels while introducing a simple phase difference between sinusoidal components of the signal. The adaptive filter responds by forming a transfer function equivalent to that of a narrowband filter centered at the frequency of the narrowband signal. The output $\mathrm{y}(\mathrm{n})$ of the adaptive filter in the ALE is an estimate of the noise free input signal. Delay $\Delta$ is selected such that $\Delta$ is longer than $\tau_{\mathrm{d}}(\mathrm{BB})$ i.e. correlation length of the broadband noise is smaller than correlation length of narrowband signal i.e. $\tau_{\mathrm{d}}(\mathrm{NB})$, beyond these lags, the respective correlations die out quickly.

$$
\text { i.e } \tau_{\mathrm{d}}(\mathrm{BB})<\operatorname{Delay}(\Delta)<\tau_{\mathrm{d}}(\mathrm{NB})
$$

The ALE is simulated using LMS block of Simulink® as shown in Figure 7. The reference signal is a delayed version of primary signal therefore $X_{o}(t)$ is given to the desired port of LMS block and is delayed by $\Delta$ and further given to the input port of LMS block of simulink. NQR signal model $S_{o}(t)$ is a narrowband signal while noise $W_{o}(t)$ is a broadband signal. The delaying of signal decorrelates the noise component. Thus, the error i.e the difference between $X_{o}(t)$ and ALE output is only noise. The delay is chosen according to equation no.4, i.e. delay $96<\Delta<360$ (for NQR signal model as noise bandwidth is $30 \mathrm{kHz}$ and signal is assumed to be $8 \mathrm{kHz}$, fs $/ 30=96$, fs $/ 8=360$ ). The other parameters such as Length of filter $\mathrm{L}=256$ and step size $\mu=0.1$ are kept the same as that for ANC. The error is employed to update the filter weights such that ALE output is close to $S_{o}(t)$.

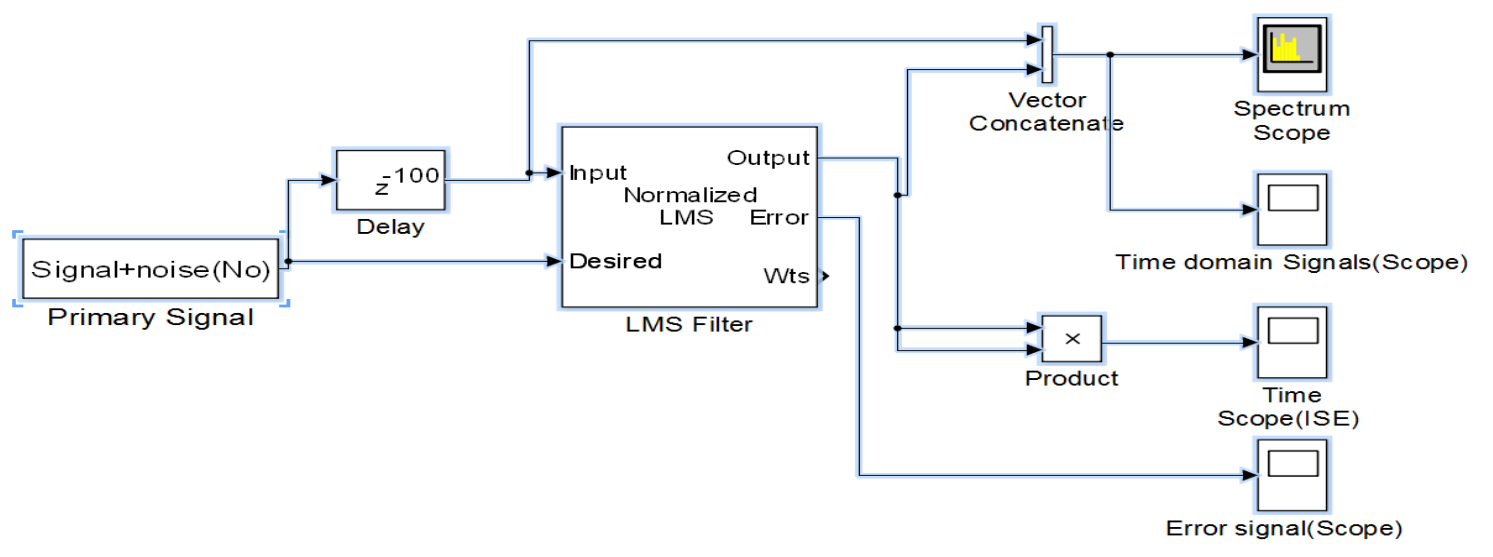

Figure 7. ALE implemented in Simulink

The real time ${ }^{14} \mathrm{~N}$ NQR signal from $\mathrm{NaNO}_{2}$ acquired from NQR spectrometer is shown in Figure 8. Due to low SNR of a single FID, it is enhanced by accumulation [12]. The results of applying ALE algorithm to real time signal are shown in Figure 8 and the corresponding power spectrum is shown in Figure 9. Figure 10 shows Instantaneous Square Error (ISE) when ALE is applied to real NQR FID signal. The results show that ISE converges within $1 \mathrm{~ms}$. The ANC algorithm is not applied to the real NQR as noise cannot be acquired separately from NQR signal. 


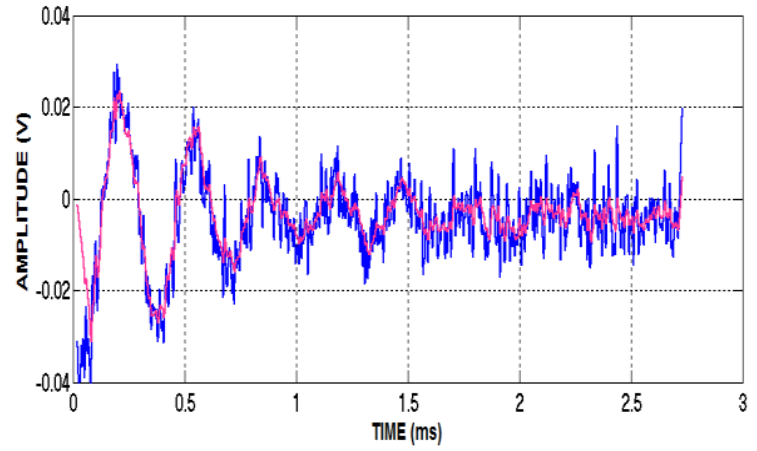

Figure 8. NQR signal of $\mathrm{NaNO}_{2}$ at input (blue) and output (pink) of ALE

The acquired NQR signal comes through $30 \mathrm{kHz}$ filter thus delay for ALE is decided by the cutoff frequency $(30 \mathrm{kHz})$ and sampling frequency $(2.875 \mathrm{MHz})$ which is 96 [11].

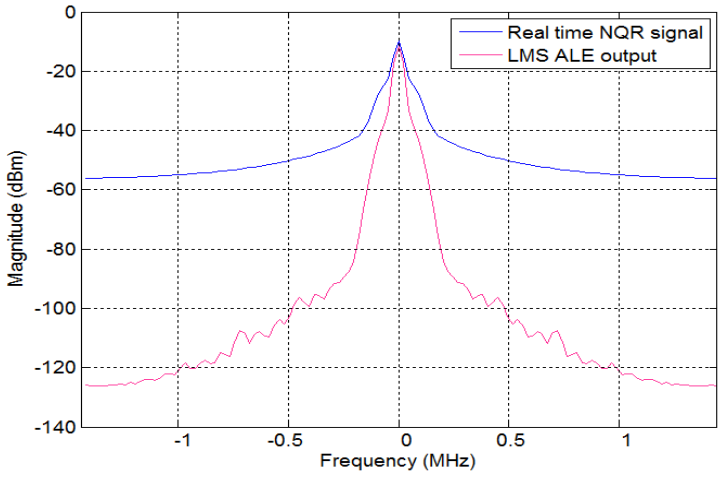

Figure 9. Power spectrum of real time NQR signal of $\mathrm{NaNO}_{2}$ and output of ALE

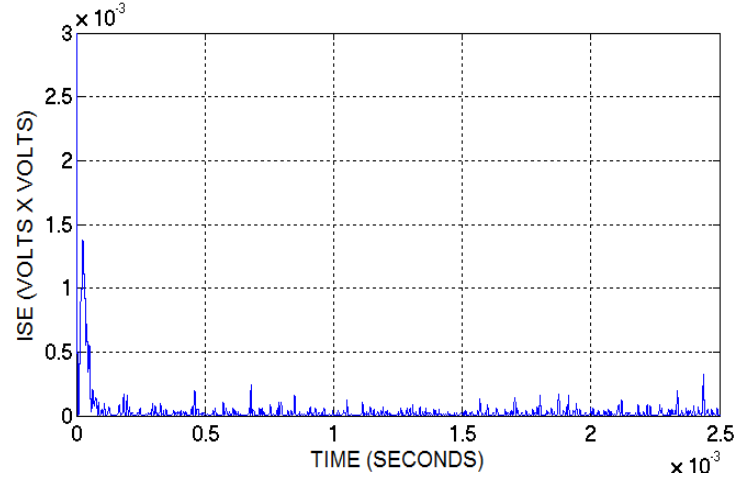

Figure 10. ISE for real time NQR as input to ALE

\section{SUMMARY AND FUTURE SCOPE}

The low signal to noise ratio (SNR) in NQR spectrometer has been observed to present certain impending challenges pertaining to high interferences in these types of detection techniques. Therefore, the current research has developed an improved NQR/NMR spectrometer based on FPGA by incorporating high speed ADCs, RF power amplifier, pre amplifier, cross diodes, quarter wave transformer and band pass filter. The incorporated permanent magnet achieved a uniform field NMR signal of proton and deuterium from $\mathrm{H}_{2} \mathrm{O}$ and $\mathrm{D}_{2} \mathrm{O}$ respectively. Also, to improve the SNR adaptive filter algorithms such as ALE was applied in real time signal to the NQR signal model. For further research, this NQR/NMR spectrometer can be further developed into a miniature spectrometer through the integration of RF amplifier, reciever module and FPGA module on one board. In this manner, the explosive detection and mine detection mechanisms of NQR/NMR experiments can be enhanced and result in more productive performance.

\section{REFERENCES}

[1] A. N. Garrowway, et al., "Remote Sensing by Nuclear Quadrupole Resonance," IEEE trans. Geoscience and remote sensing, vol. 39, no. 6, 2001.

[2] Gudmuundson E., et al., "NQR based Explosive detection-An Overview," $9^{\text {th }}$ International symposium on signal Circuits and systems (ISSCS 2009), IASI, Romania, 2009.

[3] R. Fukushima, "Experimental Pulse NMR," Nuts and Bolts approach.

[4] P. Hemnani, et al., "Development of low frequency FPGA based NQ/NMR spectrometer," Global journal of Researches in Engineering: F, Electrical and Electronics engineering, vol. 16, no. 2, 2016.

[5] P. Hemnani, et al., "FPGA based RF pulse generator for NQR/NMR spectrometer," in International Conference on Advances in Computing and Communications, Cochin, September 2016, published in Proceedings Computer Science, vol. 93, pp. 161-168, 2016. 
[6] T. C. Farrar and E. Becker, "Pulse and Fourier Transform NMR: Introduction to theory and Methods," Academic Press.

[7] J. Seliger and V. Zagar, "New methods for detection of ${ }^{14} \mathrm{~N} N \mathrm{NQ}$ frequencies," Applied magnetic resonance, vol. 43, no. 4, pp. 469-484, 2012.

[8] T. N. Rudakov, "Detection of Explosives by NQR method: Main aspects for Export Security," series 1874-6500, NATO Science for peace and security, Springer, pp. 111-138, 2009.

[9] R. Xie, et al., "De-noising methods for NMR logging echo signals based on wavelet transform," J. Geophys. Eng., vol. 11, 2014.

[10] T. Yang, et al., "NQR Signal Processing Based on Multi-stage Wiener Filter," Symposium on Security Detection and Information, Procedia Engineering, vol. 7, pp. 229-234, 2010.

[11] J. R. Mohammed, et al., "An Efficient Adaptive Noise Cancellation Scheme Using ALE and NLMS Filters," International Journal of Electrical and Computer Engineering (IJECE), vol. 2, no. 3, pp. 325 332, 2012.

[12] R. M. Ramli, "A Review of Adaptive Line Enhancers for Noise Cancellation," Australian Journal of Basic \& Applied Sciences, vol. 6, no. 6, pp. 337, 2012. 